\title{
ILCEA
}

Revue de l'Institut des langues et cultures

d'Europe, Amérique, Afrique, Asie et Australie

$20 \mid 2014$

Le conte : d'un art à l'autre

\section{« Mais il voulait tout d'abord vivre des choses et acquérir une expérience qui lui serait utile plus tard, à lui qui allait devenir roi » (comparaison de «Prince Bajaja » de Božena Němcová avec « Jean de fer » des frères Grimm)}

"But first of all he wanted to live a few things and gather some experience which, in the future, would be of use to him, the one that was to become king" (a Comparison between "Prince Bajaja" by Božena Němcová and "Iron John" by the Grimm Brothers)

„Er wollte nur zuvor Erfahrungen sammeln, die ihm als dem zukünftigen Könige nützlich sein könnten“ („Prinz Bajaja“ von Božena Němcová im Vergleich mit „Der Eisenhans“ der Brüder Grimm)

Lubomír Sůva

\section{OpenEdition}

\section{Édition électronique}

URL : http://journals.openedition.org/ilcea/2613

DOI : 10.4000/ilcea.2613

ISSN : 2101-0609

Éditeur

UGA Éditions/Université Grenoble Alpes

\section{Édition imprimée}

ISBN : 978-2-84310-290-5

ISSN : $1639-6073$

\section{Référence électronique}

Lubomír Sůva, « « Mais il voulait tout d'abord vivre des choses et acquérir une expérience qui lui serait utile plus tard, à lui qui allait devenir roi » (comparaison de «Prince Bajaja » de Božena Němcová avec " Jean de fer » des frères Grimm) », ILCEA [Online], 20 | 2014, Online erschienen am: 08 Dezember 2014, abgerufen am 10 Dezember 2020. URL : http://journals.openedition.org/ilcea/2613 ; DOI : https://doi.org/10.4000/ilcea.2613 


\section{«Mais il voulait tout d'abord vivre des choses et acquérir une expérience qui lui serait utile plus tard, à lui qui allait devenir roi » (comparaison de « Prince Bajaja » de Božena Němcová avec « Jean de fer » des frères Grimm)}

"But first of all he wanted to live a few things and gather some experience which, in the future, would be of use to him, the one that was to become king" (a Comparison between "Prince Bajaja" by Božena Němcová and "Iron John" by the Grimm Brothers)

„Er wollte nur zuvor Erfahrungen sammeln, die ihm als dem zukünftigen Könige nützlich sein könnten“ („Prinz Bajaja“ von Božena Němcová im Vergleich mit „Der Eisenhans“ der Brüder Grimm)

Lubomír Sůva

Der klassische Märchenschatz aus dem 19. Jahrhundert reflektiert nicht den wirklichen Zustand der Folklore, sondern stellt eine Art ihrer Transformation dar. Die Märchenforschung der Brüder Grimm (Jacob: 1785-1863, Wilhelm: 1786-1859) bedeutete in dieser Hinsicht einen Wendepunkt: Ihre Theorie, der zufolge die Märchen Fragmente der alten (nationalen) Poesie der vorchristlichen Zeit seien, war sowohl innerhalb als auch außerhalb der deutschsprachigen Länder einflussreich. Die Grimms haben dabei auf die Märchenforschung, -sammlung und -bearbeitung nicht nur auf dem theoretischen Feld gewirkt, sondern mit ihren Kinder- und Hausmärchen ${ }^{1}$ auch ein praktisches Beispiel gegeben, wie die romantische Sammeltätigkeit der Prosafolklore aussehen sollte. Heinz 
Rölleke fasst dies in seinem Nachwort zu der von ihm herausgegebenen Edition der dritten Ausgabe der KHM aus dem Jahre 1837 wie folgt zusammen:

Die Grimms waren auf der Suche nach dem echten alten Volksmärchenton und -repertoire: Beiden glaubten sie in jedem Stadium ihrer Arbeit näherzukommen - in Wirklichkeit aber schufen sie dabei eine neue literarische Gattung, nämlich das Grimmsche Buchmärchen mit seinem kanonbildenden Repertoire und seinem unverwechselbaren und fortan für viele Märchenerzähler und -sammler vorbildlichen Ton. (Grimm/Rölleke 1985: 1159)

2 Auch die Tschechen, die damals eine der Nationen in der österreichischen Vielvölkermonarchie bildeten und sich in der ersten Hälfte des 19. Jahrhunderts von der vorherrschenden deutschen Kultur zu emanzipieren bemühten, waren von diesem Konzept begeistert und folgten ihm. Obwohl die Rezeption und unmittelbare Wirkung der KHM in der tschechischen Literatur erst langsam einsetzte, lassen sich die Spuren der vom Zeitgeist überlieferten grimmschen Gedanken auch im Werk derjenigen Autoren finden, die (aller Wahrscheinlichkeit nach) von der Sammlung der Grimms nur indirekt beeinflusst worden waren ${ }^{2}$. Dies gilt insbesondere für das Werk der ersten bedeutenden tschechischen Schriftstellerin Božena Němcová (1820-1862), die ihre Märchen in den Jahren 1845-1847 unter dem Titel České národní báchorky a pověsti (Tschechische Volksmärchen- und Sagen $)^{3}$ in insgesamt sieben Bänden herausgegeben hat. Němcová, deren Märchen bei den Tschechen ähnliche Popularität erlangten wie die KHM bei den Deutschen, setzte sich aktiv für die Nationalbewegung ein und ist nicht nur durch ihr Werk, sondern auch durch ihr nonkonformistisches und selbstbestimmtes Leben zu einer Ikone der tschechischen Geschichte geworden, u.a. für ihre kompromisslose Unterstützung der national-bürgerlichen Revolution des Jahres 1848, derentwegen sie in den 1850er Jahren unter Polizeiüberwachung stand und unter großer finanziellen Not litt ${ }^{4}$.

3 In der kritischen Rezeption ihrer Märchen wird Němcová als Autorin mit einem , ,subjektiven, novellistischen Märchenstil“ (EM 9 (1999): 1355) rezipiert und vorzugsweise für die individualisierten Figuren der jungen und selbstbewussten Frauen beachtet ${ }^{5}$. Ihre männlichen Figuren, und darunter insbesondere die Reckenfiguren, werden dagegen oft nur am Rande erwähnt oder sogar negativ kritisiert ${ }^{6}$. Ziel dieses Beitrags ist es zu zeigen - am Beispiel der Textgenese von Němcovás Reckenmärchen „Prinz Bajaja“ ${ }^{\text {“7 }}$, dass diese weithin akzeptierte These nicht uneingeschränkt für alle von Němcovás Märchen mit männlichen Helden zutreffend ist. Innerhalb der Untersuchung werden die Stoffgeschichte und die Entwicklung der Heldenfigur von „Prinz Bajaja“ in den drei chronologisch vorausgehenden Reckenmärchen „O třech zaklených psích“ („Von den drei verwunschenen Hunden“, 1845), „Šternberk“ (1846) und „Divotvorný meč („Das Wunderschwert", 1846) nachverfolgt. Des Weiteren werden die besprochenen Märchen Němcovás mit den motivverwandten Märchentypen der Brüder Grimm verglichen und es werden Unterschiede und Ähnlichkeiten in den beiden Textkorpora analysiert. Darüber hinaus wird nach den möglichen Ursachen von Němcovás Popularität gefragt, derer sich - trotz der Fachkritik - die weiblichen Helden, wie auch die männlichen Recken bis heute erfreuen.

Das erste Märchen, dessen Elemente später in „Bajaja“ integriert worden sind, heißt „Von den drei verwunschenen Hunden“ (veröffentlicht am 20. 6. 1845, im ersten Band der Tschechischen Volksmärchen) ${ }^{8}$. Aus diesem Märchen, das grob dem ATU Typ Nr. 300 The Dragon-Slayer (Drachentöter) entspricht, hat Němcová für „Bajaja“ die Episode des Drachenkampfs und einige Merkmale der Heldenfigur entnommen und aufgewertet. 
Unterschiede zwischen Němcová und den Grimms werden am Beispiel der Drachenkampf-Episode aus den „Drei Hunden“ sichtbar, die mit einer ähnlich gestalteten Situation aus KHM 60 (1819-1857) „Die zwei Brüder“ verglichen wird. Genauso wie bei den Grimms erfolgt bei Němcová nach einem siegreichen Drachenkampf ein regressiver Prozess: Der Held hat eine Prinzessin zwar vom Drachen befreit, heiratet sie aber nicht. Dieser regressive Prozess spielt sich bei Němcová jedoch nicht auf der metaphorischen Ebene des Textes ab, deren Bezug zur psychologischen Entwicklung des Helden vom Leser selbst hergestellt werden muss - in KHM 60 wird der Held von einem falschen Helden umgebracht, mithilfe seiner treuen Tiere wiederbelebt und gewinnt erst danach die Prinzessin wieder -, sondern wird durch die persönliche Unsicherheit des Helden motiviert. Němcovás Held Vítek findet für seinen Abschied von der Prinzessin den Grund, dass ihn ein Versprechen binde, das er nicht brechen dürfe.

Fast bedauerte es Vitek, so stolz gewesen und mit der Jungfrau nicht gegangen zu sein; denn ihn band kein Versprechen, er wollte nur zuvor in der Welt Erfahrungen sammeln, die ihm als dem zukünftigen Könige nützlich sein könnten. (Němcová/ Waldau 1860: 481)

Der Selbstzweifel, die introspektive Art der Handlung sowie soziales Engagement sind typische Eigenschaften von Němcovás Figuren. In den „Drei Hunden“ ist dieses „Etwaswerden-wollen"-Motiv in der Heldencharakteristik zwar schon präsent, in der Gesamtstruktur hat es sich jedoch noch nicht durchgesetzt und bleibt unter der motivischen Fülle eines wohl mit mehreren unterschiedlichen Varianten kontaminierten Märchens verborgen ${ }^{10}$. Erst in den weiteren Texten ist es Němcová gelungen, diese Motivik auszuarbeiten und zu einem Markenzeichen ihres Werkes zu machen.

7 Eine Weiterentwicklung des Recken-Stoffes lässt sich im Märchen Das Wunderschwert verfolgen (veröffentlicht ungefähr ein Jahr später, am 26. 5. 1846, im dritten Band der Tschechischen Volksmärchen) ${ }^{11}$. Das Märchen basiert ebenso wie „Bajaja“ auf dem ATU Typ Nr. 314 Goldener. Im Hauptteil - ein Held dient getarnt am Hofe eines Königs und gewinnt mithilfe eines Helfers eine Prinzessin zur Braut - weist es Übereinstimmungen mit dem KHM 136 (1815-1843) „De wilde Mann“ (KHM 136 (1850-1857)) „Der Eisenhans“ auf. In „Bajaja“ wurde aus dem „Wunderschwert“ die komplette Fabel mit Ausnahme der Eingangsepisode des Heldendienstes bei einer Hexe übernommen.

Ein Sondermerkmal von Němcovás Variante stellt im Vergleich zu „Bajaja“ sowie zu den beiden grimmschen Märchen die Figur des magischen Helfers dar. Er erscheint in Gestalt eines Zauberpferdes, das sich als die verzauberte Mutter des Helden Vojtěch ausgibt, die - wie erklärt wird - auf der Erde das Fegefeuer abbüßen muss ${ }^{12}$. Mithilfe der Mutter wird Vojtěch von Zuhause in die Märchenwelt befördert und seine Schritte werden von ihr einem positiven Ausgang entgegen gelenkt, so dass er am Ende seine Braut, eine Prinzessin namens Milada, samt einem Königreich gewinnt ${ }^{13}$. Die fortwährende Betreuung des Helden durch die Mutter führt aber zugleich dazu, dass dem Helden eine Statistenrolle zwischen zwei Frauen zugewiesen und die Mutter zur Konkurrentin der zu gewinnenden weiblichen Figur wird. Infolgedessen und durch Einbringung von Erwachsenenpsychologie kommt es dazu, dass das Märchen etwas an naiver Märchenhaftigkeit im grimmschen Sinne entbehrt.

Vojtěch ist gekommen, hat den grünen Kranz entzwei gehackt, konnte aber nicht anders, als kurz vor dem Gerüst, auf dem Milada saß, anzuhalten und willenlos das ansteckende Feuer ihrer Augen in sich einzusaugen. Alle standen auf und schrien; und auch Milada wurde vor Freude mal blass, mal errötete sie, denn sie dachte, der 
goldene Reiter würde dieses Mal bei ihr stehen bleiben. (Němcová 1956, Sv. 1: 223, Übersetzung des Verfassers) ${ }^{14}$ glücklich sein kann, wenn die Schwiegermutter endgültig aus seinem Leben verschwindet, die gesteigerte Liebesqual der Hauptfigur sowie einige Kompositionsunstimmigkeiten - durch Eingliederung der Eingangsepisode, in welcher der Held einen Fürsten erlöst, der in ein Pferd verwandelt worden war, wird die Zahl der verzauberten und sprechenden Pferde und dementsprechend auch der potenziellen Helfer bzw. Helden für kurze Zeit verdoppelt ${ }^{15}$ - machen im „Wunderschwert“ die Hauptunterschiede zu der regelmäßigen Märchenstilisierung im grimmschen Sinne aus. Erst in „Bajaja“, welches eine weitere Bearbeitung des Goldener-Typus darbietet, ist es Němcová gelungen, alle bisher besprochenen Motive und Themen zu vereinen und eine neue und originelle Form des tschechischen „Volksmärchens“ zu schaffen.

Märchen „Prinz Bajaja“ entstammt, wie angedeutet, der Spätphase von Němcovás Märchenschaffen und stellt somit einen der letzten Versuche der Autorin dar, zu Form und Stil des tschechischen „Volksmärchens“ beizutragen (es wurde ungefähr ein Jahr nach dem „Wunderschwert“ und ungefähr zwei Jahre nach den „Drei Hunden“, am 11.03.1847 im sechsten Band der Tschechischen Volksmärchen veröffentlicht). Die kritische Rezeption des Märchens fällt überwiegend negativ aus. Václav Tille (1867-1937), ein bedeutender tschechischer Folklorist und Literaturkritiker, Herausgeber des tschechischen Märchenkatalogs und Němcová-Biograph ${ }^{16}$, sah zwischen dem „Wunderschwert“ und „Bajaja“ keinen gravierenden Unterschied und hielt Němcová eine fehlgeschlagene kreative Eigenleistung an beiden Märchen vor:

Die zwei Geschichten über einen Recken mit einem Zauberpferd [d. h. „Bajaja“ und „Divotvorný meč“], der getarnt bei einem König dient und im Turnier bzw. durch Kriegshilfe eine Prinzessin zur Braut gewinnt, reproduzieren nur sehr schwach den ursprünglichen Märchenstoff und behelfen sich dabei mit diversen Ornamenten und stofffremden Zutaten. (Tille 1909: 144, Übersetzung des Verfassers) ${ }^{17}$

11 Ähnlich urteilt siebzig Jahre später Gudrun Langer (1947-2004), eine herausragende Kennerin tschechischer Märchen des 19. Jahrhunderts, indem sie Němcová mangelnde Kreativität und Fahrlässigkeit vorhält, in deren Folge „Bajaja“ kompliziert und unübersichtlich sei:

Die literarische Eigenleistung an den vier übrigen Beiträgen des Bandes [d.h. des Bandes 6] dürfte gering sein. Die beiden Zaubermärchen „Ptáčí [sic!] hlava a srdce“ und „Princ Bajaja“ zeichnen sich durch ausgesprochen vielschichtige und komplizierte Handlungsverläufe aus. (Langer 1979: 341)

Die kritischen Einwände sind bei genauerem Blick auf die Märchenstruktur schwer zu verstehen. Anders als von Langer und Tille vermutet, geht es im Fall von „Bajaja“ um einen komplexen und künstlerisch anspruchsvollen Text, in dem Elemente aus allen besprochenen Märchen integriert und $\mathrm{zu}$ einer neuen Einheit verbunden werden ${ }^{18}$. Zusammen mit dem Hauptteil des Märchens, der aus dem "Wunderschwert" übernommen wurde, wird aus den „Drei Hunden“ die Drachenkampf-Episode interpoliert und auf der Ebene der Heldenfigur das „Etwas-werden-wollen“-Motiv zur treibenden Kraft der Geschichte gemacht. Anders als im „Wunderschwert" steht die Mutter-Sohn Beziehung nicht länger im Zentrum der Aufmerksamkeit, sondern ist zur Rahmengeschichte geworden; und anders als in den beiden erwähnten Märchen, in denen die Kindheit des Helden außer Acht gelassen wurde, kann man in „Bajaja“ die Geschichte des Helden von seiner Geburt an über dessen Initiationskonflikt mit der Mutter bis zum

ILCEA, 20 | 2014 
Erwachsenwerden verfolgen. In seiner Form steht „Bajaja“ dem grimmschen „Volksmärchen“ sehr nahe. Es weist auf der Kompositions- und Stilebene eine klare Einheitlichkeit auf und ist einem zentralen Thema, dem Reifungsprozess des Helden, untergeordnet.

Der Held, der erstgeborene Sohn eines Königs, wurde von seiner Mutter für den Zweitgeborenen erklärt und seiner Erbschaft entzogen. Damit will sich der Held nicht abfinden und entscheidet sich, sein Zuhause zu verlassen. Er vertraut sich seinem Pferd an, das ihm mit menschlicher Stimme antwortet und Unterstützung verspricht. Auf seinen Rat hin gibt sich der Held als stummer und körperlich entstellter Gartenjunge aus und dient mithilfe dieser Tarnidentität am Hofe eines fremden Königs. Dort verliebt er sich in die jüngste Königstochter und bekommt einen neuen Namen-Bajaja. In Gestalt eines unbekannten Ritters und mit der Hilfe des Pferdes tötet er drei Drachen, hilft dem König im Krieg und besiegt alle seine Konkurrenten im Turnier. Nach der Heirat mit der Prinzessin und nach der Enthüllung seiner wahren Identität kehrt er nach Hause zurück und übernimmt die Erbschaft, die ihm am Anfang verweigert wurde.

Die Komposition von „Bajaja“ ist im Sinne von V. J. Propps Morphologie des Märchens regelmäßig und streng nach dem Dreizahl-Prinzip aufgebaut: Das aus den „Drei Hunden“ interpolierte Drachenkampfmotiv verbindet sich mit den zwei anderen Reckenproben in eine Dreiereinheit (Drachenkampf, Krieg, Turnier), bei den Zahlen in der DrachenkampfEpisode werden die Dreifachen beachtet (drei Drachen, die jeweils 9, 18 und 27 Köpfe haben). Gegenüber dem „Wunderschwert“ wurde zwar die Anzahl der Nebenfiguren nicht weiter reduziert, es herrscht unter ihnen jedoch eine klare Hierarchie, in welcher der Held eine dominante Rolle spielt (auf die Verdoppelung der potenziellen Helden bzw. Helfer wurde verzichtet, die Entwicklung der Geschichte ist komplett an die Aktivität des Helden gebunden). Der Held - anders als die Namensvettern und Helden der vorigen Märchen Vítek und Vojtěch - ist von adliger Herkunft und trägt den märchenhaften sprechenden Namen Bajaja, den er im Dienst am Hof eines fremden Königs für seine ständig wiederholte Antwort „bajaja“ (,ja, ja, ja“) erhalten hat ${ }^{19}$. Alle Figuren wie Drachen, Könige, Königstöchter, aber auch die Requisiten und Kulissen (sowie die zu ihrer Darstellung verwendeten Farben) sind stereotypisch märchenhaft, und die Magie des Märchens funktioniert nach einem „natürlichen“ Wunder-Prinzip im grimmschen Sinne, was in den vorigen Märchen, die von der Feenmärchentradition bzw. von der Tradition des Volksbuches beeinflusst sind, noch nicht der Fall war. Anders als die Erscheinung des Zauberpferdes und der Zauberhunde in den beiden vorigen Märchen wird in „Bajaja“ das Erscheinen des magischen Helfers nicht mehr durch Verzauberung erklärt: Er erscheint (und verschwindet) - genauso wie die Zaubergaben - „von allein“, aus dem magischen Wesen der Märchenwelt.

Neben den Gemeinsamkeiten auf formaler Ebene lassen sich im Fall von „Bajaja“ zwischen den Grimms und Němcová auch handlungstechnische und thematische Parallelen ausmachen, und zwar (wie beim „Wunderschwert“) zu KHM 136 (1815-1843) „De wilde Mann“/KHM 136 (1850-1857) „Der Eisenhans“"20. Ähnlich wie bei Němcová vollzog sich bei den Grimms im Laufe der Zeit ein Umarbeitungsprozess, in dem eine plattdeutsche gegen eine hochdeutsche, mit mehreren Varianten kontaminierte Fassung, ausgetauscht wurde. Die hochdeutsche Fassung ist chronologisch ungefähr drei Jahre später als Němcovás Märchen veröffentlicht worden - sie bietet somit eine seltene Gelegenheit, eine frühere Variante von Němcová mit einer späteren Variante der Grimms zu vergleichen - und liegt dem „Bajaja“-Märchen deutlich näher als die gestrichene 
plattdeutsche Version, die im Zuge der Nachahmung einer authentischen Erzählung „aus dem Volksmunde" einige drastische und groteske Details aufwies sowie über äußerst spärliche Beschreibungen verfügte (u. a. blieb in der plattdeutschen Version die Beschreibung des „wilden Mannes“ völlig aus) ${ }^{21}$. Ein getarnter Held mit einer Doppelidentität und somit auch das Aschenputtel-Thema ist zwar beiden (bzw. allen drei) Texten gemeinsam ${ }^{22}$, dennoch lassen sich trotz der Stil-, Thema- und Sujetannäherungen zwischen „Eisenhans“ und „Bajaja“ Unterschiede feststellen, die auch sonst für Němcová und die Grimms typisch sind.

Abgesehen von der „mythologischen“ Basis, die die Grimms im Märchen zu entdecken vermuteten und die bei Němcová nicht thematisiert wurde, besteht der Hauptunterschied zwischen den beiden Texten in der Charakteristik des Helden und den Prüfungen, die er bestehen muss ${ }^{23}$. Bei den Grimms wird - wie in den KHM häufiger - die exemplarische Ebene der Geschichte in den Vordergrund gerückt. Die zentrale Rolle in der Entwicklung des Helden spielt ein Tabubruch, den er nach seinem Weggang von Zuhause beim Eisenhans begeht, einem dämonischen Wesen, das allein in einem Zauberwald lebt, halb wie ein Tier, halb wie ein Mann aussieht und den Menschen gefährlich ist. Nachdem der Held dreimal in einer Geduldsprobe scheitert - er lässt seine Finger und sein Haar in einem Brunnen vergolden, den er bewachen sollte, damit nichts hineinfällt - wird er der Obhut des Eisenhanses entzogen und in die Welt geschickt, um zu erfahren „wie die Armut thut" (Grimm 1850: 285). Der Eisenhans übernimmt in dem Märchen die Rolle eines Erziehers und zudem eines gefährlichen Geistes, der bestraft und belohnt. Erst durch die von ihm auferlegte Strafe kommt es beim Helden zu einem Wendepunkt und er lernt langsam und mühsam, nicht mehr das unerzogene Kind vom Anfang der Geschichte zu sein, das die Konsequenzen seiner Taten nicht tragen will ${ }^{24}$, sondern ein anständiger Mensch (im grimmschen Sinne), der sein Brot zu verdienen weiß. Der Fleiß und die Bescheidenheit, die bei den Grimms immer die moralischen Maximen sind - und die der Held, genauso wie die Arbeit in der Küche, wiederum mit der Figur des Aschenputtels teilt-, werden in diesem Märchen um eine maskuline Ebene der Reckenaufgaben bereichert - wie Bajaja hilft auch der grimmsche Held in Gestalt eines unbekannten Ritters einem König im Krieg und kämpft im Turnier -, deren Bewältigung schließlich aus dem Helden einen reifen Mann und König macht. Zusammenfassend lässt sich der Protagonist des „Eisenhans“-Märchens als ein romantischer, typisch grimmscher naivpositiver Held beschreiben, der trotz seiner adligen Herkunft eine schlichte und edle "volkstümliche“ Basis in sich trägt ${ }^{25}$, dessen Inneres fast ausschließlich in das Äußere, d. h. in die Handlung verlagert wird und in dessen Taten sich neben der sichtbaren Dimension auch eine Botschaft über den aktuellen Zustand seiner menschlichen Entwicklung widerspiegelt ${ }^{26}$.

Bei Němcová dagegen ist nicht das Exemplarische, sondern das Innere des Helden akzentuiert. Die Schlüsselsituation des Märchens ist, anders als bei den Grimms, nicht durch einen Tabubruch, sondern durch den Initiationskonflikt des Helden mit der Mutter definiert, welcher durch die innere Ambition des Helden - das verinnerlichte „Etwaswerden-wollen“-Motiv aus den „Drei Hunden“ - ausgelöst wird. Anders als Vojtěch im „Wunderschwert" lehnt Bajaja die ihm angebotene Fürsorge der Mutter ab, er möchte nicht seinem untüchtigen Bruder, der „immer um die Mutter herum“ (Němcová/Jech 1961b: 157) war, ähnlich sein - auch nicht, wenn es bedeutet, dass ihm die Erb- und Nachfolgerschaft entzogen wird -, und entscheidet sich für einen Weggang von Zuhause 
und für die Suche nach seiner Lebensaufgabe, die als unbestimmtes, erahntes Ziel vor ihm steht.

Das traf ihn hart; es machte ihm das Leben daheim so schwer, daß er in die Welt hinauswollte. Eines Tages vertraute er sein Leid dem kleinen Pferdchen an und sagte ihm, daß er fort möchte. (Němcová/Jech 1961b: 157)

Bajaja ist ein zweifelnder Held, dessen Gefühlslandschaft und Entscheidungsprozesse dem Leser zugänglich sind. Die mythologisch-erzieherische Beziehung des Helden und des Dämons bei den Grimms wird bei Němcová durch eine märchenhaft-lyrische Beziehung des Helden mit seinem treuen Zauberpferd ersetzt, mit dem er alle seine Abenteuer (in seinem Sattel) eng verbunden erlebt und mit dem er sich in einer freundlichen und vertraulichen Weise über alles berät. Auch $\mathrm{zu}$ den Menschen baut Bajaja seine Beziehungen auf einer Vertrauens-, Freundschafts- und schließlich - zu der jüngsten Prinzessin Slavěna - Liebesbasis auf und ist dabei, obwohl in „Bajaja“ eine für Němcová sonst typische gesellschaftskritische Ebene fehlt, allen ihren anderen gesellschaftlich engagierten Helden ähnlich ${ }^{27}$. Das körperliche Handicap des Helden, ein Schweigegebot, das er auf Rat seines magischen Helfers im Dienste des Königs einhält, verwandelt sich bei Němcová in eine Metapher für Bajajas Reifungsprozess, die verglichen mit den Grimms - da mit der Unmöglichkeit einer Selbstartikulation verbunden - an innerlicher Eindringlichkeit gewinnt ${ }^{28}$. Das Tabu, das mit der Liebes- und Identitätsqual des Helden verbunden wird, löst sich am Ende des Märchens von allein auf, wenn Bajaja es endlich wagt, seine inzwischen gewonnene Braut anzusprechen und sich zu seiner Liebe zu ihr und zu den Taten, die er als unbekannter Ritter verrichtet hat, zu bekennen.

„Zürnst du deinem Bräutigam, daß du dich vor ihm verbirgst?“ fragte Bajaja. „Warum fragst du mich danach, du bist doch nicht mein Bräutigam“, erwiderte flüsternd Slavěna. „Ich bin es, Jungfrau, vor dir steht der stumme Bajaja, der dir Kränzlein gewunden hat, der dich und deine Schwestern vor dem Tode errettete und deinem Vater im Kriege half. Ich bin dein Bräutigam." (Němcová/Jech 1961: 168)

19 Anders als bei den Grimms muss Bajaja am Ende keine andere Figur erlösen, die in der metaphorischen Sprache des Märchens für ihn selbst stünde und endgültig seine Position übernähme ${ }^{29}$. Obwohl das metaphorische Prinzip ähnlich bleibt, wird bei Bajaja wiederum das Individuelle unterstrichen: Er erlöst „nur sich selbst“, indem er seinem „Etwaswerden-wollen“ Lebenstrieb und den Ratschlägen seines magischen Helfers treu geblieben ist. Nachdem er von seinem magischen Helfer definitiv Abschied nimmt, kehrt er zurück nach Hause um seinen verstorbenen jüngeren Bruder - eine feige und bequeme Variante des Ichs, von dem er sich abgegrenzt hatte - zu ersetzen und die Rolle des Königs und Familienvaters zu übernehmen.

Zum Schluss unserer Betrachtungen lässt sich feststellen, dass Němcová an der Form ihres bekanntesten Reckenmärchens „Prinz Bajaja“ gezielt gearbeitet hat und bewusst nach einem Zaubermärchen im grimmschen Sinne strebte, in dem anders als in den Volksbüchern und in den Feenmärchen das "natürliche“ und unbegründete Wunderprinzip herrscht und dessen regelmäßige Komposition einem zentralen Thema, das sich mit der Figur des Helden und seiner Handlung verbindet, unterworfen ist. Diese Leistung ist umso bemerkenswerter, als es in der tschechischen Tradition so gut wie keine Vorbilder gab, an die Němcová sich hätte anlehnen können, d. h. sie musste sich direkt oder - was wahrscheinlicher ist - indirekt mit dem grimmschen Vorbild eines „Volksmärchens" auseinandersetzen und somit gegen die in der damaligen tschechischen Literatur geltenden Konventionen handeln. Im Vergleich zu den Grimms nimmt bei ihr 
die mythologisch-heroische und exemplarisch-erzieherische Ebene des Märchens ab und wird durch eine lyrische Beziehung des Helden zu seinem Helfer und durch die psychologisch plausible Ebene seines inneren Reifungsprozesses ersetzt. Auf Basis eines grimmschen naiv-positiven Helden, dessen Inneres fast ausschließlich in die Handlung versetzt wird, entsteht bei Němcová ein Held, dessen Handeln durch persönliche Ambition und durch Sorge um seine Mitmenschen und Geliebten motiviert wird, und zu dessen Gefühlslandschaft und Zweifeln der Leser Zugang hat ${ }^{30}$.

Genau diese Verbindung zwischen dem grimmschen romantischen „Volksmärchen“ und dem psychologischen Figurengemälde, das nicht mehr von der Romantik, sondern vom Realismus und vom Dorfroman französischer bzw. deutscher Herkunft beeinflusst wurde, mag dazu beigetragen haben, dass das Märchen trotzt halbherziger kritischen Rezeption eine große Leserpopularität erreicht hat, die sich auch in zwei (!) unterschiedlichen Filmadaptionen niederschlug ${ }^{31}$. Darüber hinaus dürfte die Popularität von „Bajaja“ auch in der Figur des getarnten Helden selbst zu suchen sein, welcher unerkannt Reckentaten verrichtet, von den Machtträgern, denen er schweigend und lächelnd zunicken muss, jedoch bloß für einen minderwertigen Idioten gehalten wird. Diese funktionelle Umarbeitung eines alten Motivs aus der Tradition der mittelalterlichen Literatur ${ }^{32}$ ließe sich im Einklang mit dem Kollektivgefühl der (nicht nur) im 19. Jahrhundert nach der Emanzipation von einer anderssprachigen Kultur strebenden und unter permanentem Bedrohungsgefühl leidenden Tschechen verstehen und in einer Figur verkörpert sehen, die in der tschechischen Kultur in Variationen, etwa in einem der bekanntesten Romane der tschechischen Literatur Osudy dobrého vojáka Švejka za světové války (Die Abenteuer des braven Soldaten Schwejk, 1921-1923), wieder aufs Neue zum Vorschein kommt.

Nachdem Němcová die Arbeit an ihrer Märchensammlung beendet hatte, widmete sie sich-besonders in den 1850er Jahren-im Einklang mit progressiven Literaturtendenzen der Zeit überwiegend dem Dorfroman und der Dorfgeschichte. Auf diesem Feld hat sie als Autorin des ersten tschechischen Romans Babička (Die Großmutter, 1855) eine prominente Position in der tschechischen Literaturgeschichte erworben. $\mathrm{Zu}$ den Paradoxien der tschechischen Literatur, die im 19. Jahrhundert kurz vor ihrem Abgang neu belebt wurde, gehört es, dass sie ihre Voraussetzungen erst nachträglich erfüllte und dass ein „echtes Volksmärchen“ im grimmschen Sinne erst fünfzehn Jahre nach Němcová, in den 1850er bzw. 1860er Jahren, der Feder von Karel Jaromír Erben (1811-1870) entsprang ${ }^{33}$. „Die besten sind die von Erben“ (Denecke/Teitge 1989: 342) urteilt Jacob Grimm in seiner handschriftlichen Anmerkung in einer von Alfred Waldau herausgegebenen tschechischen Märchenanthologie über die Texte seines Geistesgefährten ${ }^{34}$. Diese Anmerkung ist ein Zeugnis dafür, dass an der Form des tschechischen "Volksmärchens" auch nach Němcová weiter gearbeitet und seine Geschichte in Tschechien ungefähr zeitgleich wie in Deutschland, inmitten der neuen Stilströmungen, abgeschlossen wurde. 


\section{BIBLIOGRAPHIE}

DENECKE L., TEITGE I. (1989), Die Bibliothek der Brüder Grimm, Stuttgart.

Enzyklopädie des Märchens. Handwörterbuch zur historischen und vergleichenden Erzählforschung. Begründet von K. Ranke. ed. R. W. Brednich u. a., Berlin/New York, 1977-.

FILlMORE P. (1919) (Hrsg., Übers.), Czechoslovak fairy tales, New York.

GRIMM (1815), Kinder- und Hausmärchen, gesammelt durch die Brüder Grimm, Bd. 2, Berlin, 1815

(Nachdruck [...] nach dem Handexemplar des Brüder Grimm-Museum Kassel [...] in Verbindung mit U. Marquardt von H. Rölleke. Göttingen, 1986).

GRIMM (1856), Kinder- und Hausmärchen, gesammelt durch die Brüder Grimm, Bd. 3, Göttingen, 3. Aufl. 1856 (ed. H. Rölleke. Kinder- und Hausmärchen. Mit den Originalanmerkungen der Brüder Grimm, Bd. 3, Stuttgart, 2008).

GRIMM (1850), Kinder- und Hausmärchen, gesammelt durch die Brüder Grimm, Göttingen, 1850.

GRIMM \& RÖLLEKE (1985), Kinder- und Hausmärchen, gesammelt durch die Brüder Grimm, Vollständige Ausgabe auf der Grundlage der dritten Auflage (1837), Herausgegeben von Heinz Rölleke, Frankfurt am Main, 1985.

JECH J. (1961) (Hrsg.), Tschechische Volksmärchen, Berlin.

LANGER G. (1979), Das Märchen in der tschechischen Literatur von 1790 bis 1860: Studien zur Entwicklungsgeschichte des Märchens als literarische Gattung, Gießen.

NĚMcovÁ B. (1956), Národní báchorky a pověsti, Sv. 1-2, Praha.

NOvotNÝ M. (1951) (ed.), Život Boženy Němcové, Díl 1. Praha.

POLÍVKA J., „Bratří Grimmové a počátky českého pohádkosloví.“, Z dějin české literatury: Sborník statí věnovaný Jaroslavu Vlčkovi k šedesátinám od jeho spolupracovníků a žáků. Praha 1920, s. 139-142.

Poмо Media Group (2001-2012), Česko-Slovenská filmová databáze. Bajaja, <http://www.csfd.cz/ film/33863-bajaja/>. Letzter Zugriff: 28. 12. 2012.

Ромо Media Group (2001-2012), Česko-Slovenská filmová databáze. Princ Bajaja, <http:// www.csfd.cz/film/4932-princ-bajaja/>. Letzter Zugriff: 28. 12. 2012.

PROPP V. (1972), Morphologie des Märchens, München.

RÖHRICH L. (1974), Märchen und Wirklichkeit, 3. Aufl, Wiesbaden.

RÖLLEKE H. (1985), „Wo das Wünschen noch geholfen hat.“ Gesammelte Aufsätze zu den „Kinder- und Hausmärchen“ der Brüder Grimm, Bonn.

schAMSCHULA W. (1996), Geschichte der tschechischen Literatur, Bd. 2, Von der Romantik bis zum Ersten Weltkrieg, Köln.

SŮVA L., „Wäre er nicht in der Hölle gewesen, hätte er sich wie im Himmel gefühlt. ,Des Teufels rußiger Bruder` der Brüder Grimm und ,Des Teufels Schwager ' der Božena Němcová auf Basis der Helden und ihrer Helfer verglichen.“ Lox, Harlinda, Lukas, Ricarda (Hrsg.), Heimliche Helfer, 
unheimliche Begleiter. Forschungsbeiträge aus der Welt der Märchen. Krummwisch bei Kiel 2012, S. 149-170.

TILLE V., NOvotNÝ M. (1938), Božena Němcová, 4. vyd. Praha.

TILLE V. (1909), České pohádky do roku 1848, Praha.

UTHER H.-J. (2008), Handbuch zu den „Kinder- und Hausmärchen“ der Brüder Grimm, Berlin/New York. UTHER H.-J., The types of International Folktales. A classification and Bibliography, Based on the System of Antti Aarne and Stith Thompson, vol. 1-3, Helsinky, 2004.

WALDAU A. (1860) (Hrsg., Übers.), Böhmisches Märchenbuch, Prag.

JECH J., „Klasická sbírka německých pohádek”, Grimm, J. a W.: Německé pohádky. Praha, 1961, s. 5-19.

\section{NOTES}

1. Die ersten zwei Bände sind in den Jahren 1812 und 1815 in Berlin erschienen, die Ausgabe letzter Hand wurde I857 in Göttingen veröffentlicht. Die Sammlung wird im Folgenden als KHM zitiert.

2. Die ersten Übersetzungen der KHM ins Tschechische erscheinen ab Mitte der 1830er Jahre; ein unmittelbarer Einfluss auf die Konzeption der Sammel- und Bearbeitungstätigkeit bzw. auf die jeweiligen Märchen der einzelnen tschechischen Autoren lässt sich ab Mitte der 1840er Jahren bei J. Malý und K. J. Erben beobachten. Cf. Jech 1961a: 18, Polívka 1920: 141-142.

3. Cf. Langer G.: „Němcová, Božena“, EM 9 (1999): 1354-1358. Alle sieben Bände sind in Prag erschienen. Die Sammlung wird im Folgenden als Tschechische Volksmärchen zitiert.

4. Zu Leben und Werk von Božena Němcová cf. Langer (wie Anm. 3), Schamschula 1996: 67-79, Tille/Novotný 1938.

5. „Ein weiteres Spezifikum Němcovás ist die Individualisierung der weiblichen Figuren.“ (EM 9 (1999) : 1356)

6. Václav Tille, Herausgeber des tschechischen Märchenkatalogs und Biograph von Němcová, urteilt zusammenfassend: „Romantické povídky o recích jsou u Němcové vesměs nejisté v rozvijení děje a spájení látkových motivů; stylem jsou trochu rozvláčné a nemají oné jistoty v kresbě figur jako povídky o ženách.“ („Die romantischen Reckenerzählungen sind bei Němcová in der Erzähtechnik und im Zusammenfügen von Märchenmotiven vorwiegend unsicher; im Stil sind sie ein bisschen langatmig und lassen die Sicherheit des Figurengemäldes vermissen, das ihre Erzählungen mit weiblichen Helden auszeichnet.") Tille 1909: 139; Übersetzung des Verfassers.

7. Das Märchen wird im Folgenden als Bajaja zitiert.

8. Das Märchen wird im Folgenden als Drei Hunde zitiert.

9. Wenn eine deutsche Übersetzung des jeweiligen Märchens vorliegt, wird nur auf Deutsch zitiert. Alle Hervorhebungen im Text stammen vom Verfasser, wenn nicht anders angegeben.

10. Das Märchen wurde schon in einer der zeitgenössischen Rezensionen des ersten Bandes von K. H. Borovský (veröffentlicht am 05.08.1845 in Česká včela) in Bezug auf kleine logische Unstimmigkeiten in der Erzählung kritisiert (cf. Novotný 1951: 150).

11. Das Märchen wird im Folgenden als Wunderschwert zitiert.

12. Es geht um ein ähnlich gestaltetes Motiv wie in den „Drei Hunden“; die magischen Helfer, drei Hunde, sind dort eigentlich drei verzauberte Fürsten, die ihre schlechten Taten wiedergutmachen müssen. Tille betrachtet das Fegefeuer-Motiv im „Wunderschwert“ als typenfremd und weist darauf durch das Ausrufezeichen hin (Tille 1909: 144). 
13. Die Gestalt eines sprechenden Pferdehelfers wurde aus einem anderen Reckenmärchen, dem Šternberk, interpoliert, das den dritten Band der Tschechischen Volksmärchen eröffnet.

14. „Vojtěch přijel, zelený věnec ve dví přeřal, ale nemoha jináč, zastavil se na okamžení pod lešením, kde Milada seděla, a svévolně nakažlivý oheň z jejích očí do sebe sál. Již to všecko vstávalo, křičelo a Milada radostí brzo se červenala, brzo bledla; neboř myslela, že tenkráte zlatý jezdec u ní zůstane."

15. Cf. Tille 1909: 144; Tilles pauschale Kritik ist jedoch nicht völlig berechtigt, denn er übersieht, dass der Märchenanfang eine wichtige strukturelle Rolle zu erfüllen hat, und dass die negative Stiefmutter-Stiefsohn-Beziehung des Eingangsteils sich als Strukturopposition zum positiven Mutter-Sohn-Verhältnis des Märchenhauptteils auslegen lässt.

16. Seine eigenen Märchen hat Tille unter dem Pseudonym Václav Říha veröffentlicht.

17. „Dvě povídky o rekovi s divotvorným koněm, jenž slouží nepoznán u krále a v závodech neb pomocív boji získává princeznu za choŤ, reprodukuji velmi chatrně původní pohádkové látky a vypomáhají si různými př́měsky a výzdobami.“

18. Anders als Langer und Tille urteilt der Erzähler, Übersetzer und Herausgeber tschechischer und slowakischer Märchen Parker Fillmore und weist somit auf den wahren Charakter von , Bajaja“ hin: „Nemcova's more elaborate tale, Prince Bayaya, [...] is really a mosaic of two or three simpler stories." (Fillmore 1919: o. S. (Vorrede))

19. Sprechende Namen dieser Art, die den märchenhaften Charakter des Geschehnisses unterstützen würden und im engen Zusammenhang mit grimmschen Märchentiteln wie „Rotkäppchen, Aschenputtel“ bzw. „Rumpelstilzchen“ gesehen werden könnten, kommen bei Němcová vor „Bajaja“ gar nicht vor.

20. Das Märchen wird im Folgenden als „Eisenhans“ zitiert.

21. Zur Biographie, Wirkung und Interpretation vom „Eisenhans“ und „De wilde Mann“ cf. Uther 2008: 288-292, 477. Speziell zur Textentwicklung vom „Eisenhans“ innerhalb der KHM cf. auch Rölleke 1985: 64-69.

22. Die Grimms selber merken an, dass in dem Helden des Märchens die Figur eines „männliche [n] Aschenputtel[s]“ zu entdecken sei. (Grimm 1815: Anhang XXXIX)

23. Für die Grimms ist das Märchen im Einklang mit ihrer Märchentheorie ein Überrest der (alten?) Mythologie gewesen, wie aus der zusammenfassenden Darstellung in der zweiten separaten Auflage des KHM-Anmerkungsbandes aus dem Jahre 1856 hervorgeht: „Das Märchen mag eine alte Grundlage haben und von einem höheren halbgöttlichen Wesen erzählen, das in die Gewalt eines Unterirdischen gerieth und niedrige Arbeiten verrichten mußte bis es wieder zu seiner höheren Stellung gelangte; die goldenen leuchtenden Haare weisen darauf hin.“ (Grimm 1856: 219). Němcová dagegen - obwohl sie ähnlich wie die Grimms ihre Märchen als tschechische Nationaldenkmäler bezeichnet hatte - verfügte, wie G. Langer feststellte, „über eine [...] vergleichbare mythologische Konzeption [...] nicht." (EM 9 (1999): 1356)

24. „Ach, wilder Mann, geh nicht fort, sonst bekomme ich Schläge“ ruft er, nachdem er den Eisenhans gegen das Verbot seiner Eltern aus dem Käfig losgelassen hat. (Grimm 1850: 282)

25. „Die wilden riechen kräftiger und werden ihr besser gefallen.“ (Grimm 1850: 286) sagt der Held des „Eisenhans“-Märchens über die wilden Feldblumen, die er statt der Gartenblumen der Prinzessin als Geschenk bringt. Dies steht im Einklang mit dem grimmschen romantischen Programm der Märchensammlung, in dem sie die Märchen als eine „aus sich selbst heraus“ entstehende Urpoesie deuten und sie selbst - in der Vorrede zum zweiten Band der KHM - mit den wilden Feldblumen vergleichen: „Nichts besser kann uns vertheidigen, als die Natur selber, welche gerad diese Blumen und Blätter in dieser Farbe und Gestalt hat wachsen lassen." (Grimm 1815: IX)

26. Märchen - d. h. das grimmsche Märchen - sei nach Lutz Röhrich eine „getarnte Ich-Erzählung “ (Röhrich 1974: 16), in dem „alles Äußere [...] vielmehr nur sichtbarer Ausdruck innerer Vorgänge“ ist (Röhrich 1974: 27). Cf. auch die o. a. Analyse der „Drei Hunde“ und hier besonders den Vergleich der Drachenkampf-Episode bei Němcová und bei den Grimms. 
27. Diese Ebene ist etwa, wie angedeutet, in den „Drei Hunden“ zu finden; weiter cf. insbesondere die Heldenfiguren in „Čert a Káča“ („Katja und der Teufel“), „Čertův švagr" („Des Teufels Schwager“) und „Spravedlivý Bohumil“ („Der gerechte Bohumil“). Cf. Sůva 2012: 149-170.

28. Der Held des „Eisenhans“ täuscht vor, einen grindigen Kopf zu haben.

29. „Ich bin der Eisenhans, und war in einen wilden Mann verwünscht, aber du hast mich erlöst. Alle Schätze, die ich besitze, die sollen dein Eigenthum sein“; verkündet der ehemalige Dämon am Ende der Geschichte, nachdem er als „ein stolzer König [...] mit großem Gefolge“ die Szenerie betritt. (Grimm 1850: 291)

30. Diese Tendenz zur Individualisierung der Figuren ist in den späteren Auflagen der KHM auch bei den Grimms - wie z. B. ein Vergleich von „De wilde Mann“ und „Eisenhans“ zeigt - zu beobachten, jedoch nicht in demselben Ausmaß wie bei Němcová.

31. Laut Česko-Slovenská filmová databáze (Tschecho-Slowakische Filmdatenbank) gibt es zwei filmische Bearbeitungen, die sich in ihrem Titel auf „Bajaja“ berufen : Bajaja (1950, Regie Jiří Trnka) und Princ Bajaja (1971, Regie Jiří Kachlík). Cf. POMO Media Group 2001-2012a, b. Trnkas Bearbeitung bekam weltweite Aufmerksamkeit, indem sie im Jahre 1954 mit dem Hauptpreis auf dem Filmfestival in Locarno gekrönt wurde.

32. Um für seine Sünde zu büßen, muss etwa der Held des „Predigtmärleins“ Robert der Teufel (Mitte des 13. Jahrhunderts) von Étienne de Bourbon ein Schweigegebot einhalten. Cf. Günter Dammann : „Goldener“, EM 5 (1987): 1380.

33. Erben hat philologisch auf eine ähnliche Weise wie die Grimms gearbeitet, seine Märchen als Überreste der slawischen Mythologie verstanden und sie mit ausführlichen Anmerkungen im grimmschen Stil versehen.

34. Němcová/Waldau 1860.

\section{RÉSUMÉS}

Der Beitrag untersucht die Kinder- und Hausmärchen der Brüder Grimm (Jacob: 1785-1863, Wilhelm: 1786-1859) im Vergleich mit den Märchen der ikonischen tschechischen Autorin der Moderne Božena Němcová (1820-1862). Das romantische Märchen wird hier als ein Kulturphänomen angesehen, für dessen Entwicklung die Untersuchungen der Brüder Grimm, deren zufolge die Märchen Fragmente der alten (nationalen) Poesie der vorchristlichen Zeit seien, wegweisend waren. Speziell konzentriert sich der Beitrag auf die Textgenese eines von Němcovás komplexesten und künstlerisch anspruchsvollsten Märchen „Princ Bajaja“ (ATU 314 Goldener). Im Vergleich mit dem KHM 136 „Eisenhans“ wird die bisherige negative kritische Rezeption von „Princ Bajaja“ problematisiert, indem auf die Planmäßigkeit des Aufbaus des Märchens und die Originalität in der Darstellung der Heldenfigur hingewiesen, sowie nach den Gründe der fortdauernden Popularität des Märchens im 20. und 21. Jahrhundert gefragt wird.

Le présent article analyse les Contes de l'enfance et du foyer des frères Grimm (Jacob : 1785-1863, Wilhelm : 1786-1859) en comparaison avec les contes de Božena Němcová (1820-1862), auteur emblématique tchèque de la modernité. Le conte romantique est considéré ici comme phénomène culturel, pour lequel les recherches des frères Grimm, selon qui les contes étaient des fragments de l'ancienne poésie nationale de l'époque préchrétienne, ont joué un rôle fondateur. Cet article met notamment l'accent sur la genèse textuelle du conte de fées Princ Bajaja (ATU 314 Goldener), qui est un des contes les plus complexes et les plus exigeants sur le plan 
artistique de Božena Němcová. En établissant une comparaison entre ce dernier et le conte merveilleux «Jean de fer» («Eisenhans », KHM 136), cette communication étudie la réception critique jusqu'ici défavorable de l'œuvre de Němcová en mettant en lumière la régularité de la structure du conte, et en soulignant l'originalité de la présentation du héros qui lui est propre. Elle s'interroge également sur les raisons de la popularité persistante de ce conte aux $\mathrm{xx}^{\mathrm{e}}$ et $\mathrm{xxI}^{\mathrm{e}}$ siècles.

This essay examines the Children's and Household Tales by the Grimm Brothers (Jacob: 1785-1863, Wilhelm: 1786-1859) compared with the fairy tales written by Božena Němcová (1820-1862), the iconic Czech author of modernity. The romantic fairy-tale, rather than representing the actual state of folklore of the 19th century, is here regarded as a cultural phenomenon, whose development was being fundamentally influenced by the Grimm Brothers groundbreaking theory, suggesting that fairy tales are in fact fragments of ancient (national) poetry of preChristian times. In particular, the paper focuses on the textual development of one of the most complex and artistically demanding of Němcová's tales Princ Bajaja (ATU 314 Goldener), in comparison with the Grimms' tale Nr. 136 Eisenhans (Iron John). By pointing out the clarity of the tale's composition as well as its originality in depicting a hero's character, the previous negative critical reception of Princ Bajaja is being questioned and the reasons for the continuing popularity of the tale in the 20th and 21st century are being analyzed.

\section{INDEX}

Mots-clés : contes merveilleux, romantisme, Jacob et Wilhelm Grimm, Božena Němcová, ATU 314 L'Homme aux cheveux d'or, héros, Jean de fer, Prince Bajaja

Schlüsselwörter : Märchen, Romantik, Jacob und Wilhelm Grimm, Božena Němcová, ATU 314 Goldener, Heldenfiguren, Eisenhans, Princ Bajaja

Keywords : fairy tales, romanticism, Jacob and Wilhelm Grimm, Božena Němcová, ATU 314 Goldener, hero, Iron John (Eisenhans), Prince Bajaja

\section{AUTEUR}

\section{LUBOMÍR SŮVA}

Université de Göttingen 